\title{
A Quantitative Investigation of Thermosonics
}

\author{
T. J. Barden*, D. P. Almond*, M. Morbidini* ${ }^{* *}$ P. Duffour** and \\ P. Cawley**
}

*Materials research Centre, Department of Engineering and Applied Science, University of Bath, Bath, BA2 7AY, UK

${ }^{* *}$ Research Centre for NDE, Imperial College, London, Mechanical Engineering Dept, South Kensington Campus, London, SW7 2AZ, UK

\begin{abstract}
The objectives of this study were to investigate the relationship between the ultrasonic vibrations of a specimen, with a vertical surface crack, and the thermal response. The heat required to be liberated by the defect to obtain a detectable surface temperature rise was modelled analytically for energy liberated only at the crack tip and over the sides of a crack. Experiments were carried out on a nickel based superalloy plate with a vertical surface crack. The strain around the defect and thermal image were measured simultaneously. Parameters were varied to change the strain to enable a correlation between the strain and surface temperature rise. From this the energy released from the defect was estimated using an optimisation algorithm.
\end{abstract}

\section{Introduction}

Thermosonics, or vibro-thermography, uses vibrational energy to excite a specimen, as oppose to conventional thermography for NDE that is optically stimulated. If a defect is present the vibrational energy maybe converted into heat energy at the defect and the surface temperature rise detected using a thermal imaging camera.

Several papers have been published discussing the advantages of this technique over optically stimulated thermography and have shown it is capable of detecting many types of defects in several materials [1, 2, 3, 4]. The main advantage of the heating method is that it is able to reveal defects that optical thermography cannot. These include vertical surface cracks and some types of impact damage in CFRP $[3,5]$. The use of lock in techniques has been shown to have advantages over pulse heating $[6,7]$. It has also been shown to detect some defects at deeper depths than optical thermography $[1,8]$. There are two main disadvantages of thermosonics: it is a contactive technique and a large power consumption is required by some systems to detect defects.

A quantitative study of the energy required to obtain a detectable surface temperature rise for vertical surface cracks has been conducted. The energy liberated by the defect and the strain in the bulk material have been investigated. This information will further the understanding of the heating process and should lead to the optimisation of the excitation scheme to minimise the amplitude of excitation.

\section{Analytical simulations}

There are two mechanisms by which heat is considered to be liberated by a crack. A rubbing action can cause the sides of a crack to heat up and an increased 
strain at the crack tip causes increased heating due to the thermoelastic effect. Evidence has been shown suggesting that both these cases can occur [4, 9] either independently or simultaneously. Thermosonics has previously been modelled by considering the excited crack as an embedded heat source [10] and that heat is given off over the whole area of the crack. The response at the surface was modelled only for a continuous step function and the results showed a good correlation to empirical data.

In this work the thermal response for vertical surface cracks has been modelled analytically for a finite pulse. Both heating only at the crack tip and over the area of the crack were considered. The models were used to ascertain the energy required to be generated by the defect for a detectable surface temperature rise. This was completed for nickel based superalloy, steel and aluminium. The material properties used are shown in table 1 . The models were also used for physics based curve fitting to estimate the energy liberated by actual defects.

The assumptions made for the analytical model were the crack depth was uniform, the material was homogeneous, the crack was long enough that edge effects were ignored, the radial heat flow was the same in all directions and there was adiabatic conditions at the surface.

These assumptions enabled the crack to be modelled in two dimensions. The solution for heating only at the crack tip was found by modelling the heat generated at a line source for continuous heating in infinite space, Carslaw and Jaeger [11], and is given in Eq. (1). The response for a finite pulse was found by substituting the solution into Eq. (2).

$$
\begin{aligned}
& T_{\text {cont }}(r, t)=-\frac{Q_{0}}{4 \pi k} E i\left[-\frac{r^{2}}{4 \alpha t}\right] \\
& T_{P}(t)=T_{\text {cont }}(t)-\delta(t) T_{\text {cont }}(t-\tau) \text { for } \begin{array}{ll}
\delta(t)=0 & t \leq \tau \\
\delta(t)=1 & t>\tau
\end{array}
\end{aligned}
$$

Where $T_{p}$ is the response from a pulse of $\tau$ seconds at time t a distance $r$ from the line source liberating energy at $Q_{0}$ Watts per $m$. $\alpha$ is the thermal diffusivity and $k$ is the thermal conductivity. $E i$ is the exponential integral and $\delta()$ is the Heaviside function.

It was assumed there was no heat flow across the surface and this was satisfied using the technique of images. The temperature at the surface was found to be twice as in an infinite solid.

The response for heating over the sides of the crack was found by integrating the solution for the line source over the depth of the crack, Eq.(3). The response for a finite pulse was again found by substituting Eq. (2) into Eq. (3).

$$
T_{\text {cont }}(t)=\int_{0}^{d}-\frac{Q_{0}}{2 \pi k} E i\left[-\frac{r^{2}}{4 \alpha t}\right] d z
$$

Where, $r^{2}=z^{2}+x^{2}$

$d$ is the crack depth, $x$ is the coordinate on the surface perpendicular to the crack and $z$ in the vertical coordinate. 
http://dx.doi.org/10.21611/qirt.2004.058

Table 1 : Thermal properties of materials used for the simulation

\begin{tabular}{|c|c|c|}
\hline Material & $\begin{array}{l}\text { Thermal Conductivity } \\
(\mathrm{W} / \mathrm{mK})\end{array}$ & $\begin{array}{c}\text { Thermal diffusivity } \\
\left(\mathrm{m}^{2} / \mathrm{s}\right) \times 10^{-6}\end{array}$ \\
\hline Nickel super alloy & 11 & 2.96 \\
\hline Steel & 50 & 11 \\
\hline Aluminium & 180 & 73 \\
\hline
\end{tabular}

The energy required to detect a $10 \mathrm{~mm}$ long crack excited for 0.5 seconds using a camera with a thermal sensitivity of $0.1^{\circ} \mathrm{C}$ was calculated for cracks with depths from $1 \mathrm{~mm}$ to $7 \mathrm{~mm}$. The results are illustrated in figure 1 . The heat liberated by the crack was about 0.5 to $2 \mathrm{~W}$ if the heat was assumed to be liberated at the crack tip, but less than $0.5 \mathrm{~W}$ if the energy was considered to be liberated over the sides of the crack. The difference between the two mechanisms occurs since there is always a heat source close to the surface for the heat been given off at the sides of the crack. The energy required is significantly lower than the $1000 \mathrm{~W}$ of electrical power that has been used is some previous studies [4] and suggests very little of the electrical energy is converted to heat energy at the defect.

\section{Experimental Method}

The vibration around the crack and the thermal response were investigated for a $3 \mathrm{~mm}$ thick nickel based superalloy plate with a $20 \mathrm{~mm}$ long surface crack. A strain gauge was placed on the specimen at the same position of the crack, but on the opposite face. The specimen was excited from the back face and observed from the front face. The strain was varied whilst simultaneously measuring the thermal response. Thus obtaining a relationship between the strain and the surface temperature rise. Pulse stimulation was used, since it is simpler to infer the energy liberated at the crack tip from a pulse stimulation response as oppose to lock in thermography. The pulse time was 0.5 seconds.

A Branson $20 \mathrm{KHz}$ horn was used to excite the specimens, powered by an amplifier capable of providing $400 \mathrm{~W}$. A Cedip Jade infrared camera was used to obtain the thermal images.

\section{Experimental Results}

The increase in surface temperature for a 0.5 second pulse was compared to the strain, figure 2 . The surface temperature rise was linearly related to the strain. These results tie in with work from a previous study [12]. The results show that a strain of $80-90 \mu \varepsilon$ would be required to achieve the minimum detectable surface temperature rise, about 0.1 to $0.2^{0} \mathrm{C}$, for the crack used in this study.

Using the assumptions made for the models it was possible to estimate the energy liberated at the defect for both heating mechanisms. This was achieved by physics based curve fitting. An optimisation algorithm was used, on the transient response, that fitted the crack depth and the energy liberated at the defect. This was possible since the shape of transient response curve is dependent on the depth. An example of this is shown, for both heating mechanisms, in figure 3. Both mechanisms can be made to fit the data, but with different crack depths. The curve fitting shows the model data correlates well to the empirical data. It also uses the model data to infer more information about the system that will further the understanding of the thermosonic process. 
The optimisation algorithm was used to estimate the heat liberated by the defect for all the trials, the results are shown in figure 4 . The energy liberated at the crack tip is linearly related to the strain in the bulk material. This could be inferred from figure 2 since the temperature rise is directly proportional to the energy, as shown in Eq. (1) and (3). To obtain these results the depth of the crack had to be fitted to the data. The optimisation algorithm estimated the depth to be about $286 \mu \mathrm{m}$ for heating at the crack tip only and $705 \mu \mathrm{m}$ for heating over the sides of the crack. Using these assumptions for crack depth the energy liberated at the defect was between 0.3 and $0.9 \mathrm{~W}$ for the two mechanisms. By assuming semi-infinite space this technique has over estimated the heat liberated and the crack depth. Continued development of the modelling will improve the estimation of the energy liberated at the defect.

\section{Discussion}

A good correlation between the real and model data was obtained for both heating at the crack tip only and heating over the sides of the crack. It was not possible to determine, from the curve fitting, which heating mechanism is present for this sample. If the depth was known then this could be deduced, but since further tests are being carried out on this specimen it has not been possible, as yet, to section it, enabling measurement of the crack depth.

Evidence shown in the plots of temperature rise and heat against strain, figures 2 and 4, suggest that there is a strain threshold that must be obtained for the defect to heat up. For this crack this is approximately $80 \mu \varepsilon$. If heat was liberated as a consequence of an increased strain at the crack tip one would expect a heat generation at the crack tip for any strain in the bulk material. If rubbing was present then the friction between the surfaces of the crack would have to be overcome before heat was generated and a strain threshold to activate heating would be expected. Thus these results suggest there is heating over the sides of the crack in this example.

This preliminary study has shown it should be is possible to estimate the energy liberated at a crack tip using physics based curve fitting. This work, though, has only been carried out on a single sample and more results are required to confirm the success of this approach.

\section{Conclusions}

Analytical models have been produced for pulsed heating for two heating mechanisms: heating only at the crack tip and heating over the sides of the crack. Experimental trials were conducted to ascertain the relationship between strain in the bulk material and surface temperature rise as a consequence of the crack. Physics based curve fitting was used to estimate the energy liberated at the defect and it was shown that both the temperature increase and the energy liberated at the defect were linearly related to the strain in the bulk material. This study has shown that by using physics based curve fitting and studying the strain in the material information can be gained to further the understanding of the heating mechanism of thermosonics.

\section{Acknowledgements}

This study is supported by the EPSRC, UK. The authors wish to thank Airbus UK for the use of their thermal imaging camera and the continued support from RollsRoyce, BNFL and DSTL. 


\section{REFERENCE}

[1] J Rantala, D Wu and G Busse 'NDT of polymer materials using lockin thermography with water-coupled excitation' NDT and E Int. 1996 p.43-49

[2] J Rantala, D Wu, a Salerno and G Busse 'Lock-in thermography with mechanical loss angle heating at ultrasonic frequencies' QIRT1996 p.389-393

[3] A Salerno, A dillenz, D Wu, J Rantala and G Busse' Progress in ultrasound lockin thermography' Quantitative Infrared Thermography 1998 p. 154-160

[4] L D Favro, X Han, Z Ouyang, G Sun, H Sui and R L Thomas 'Infrared imaging of defects heated by a sonic pulse' Review of Scientific instruments June 2000 p. $2418-2421$

[5] D.P. Almond, R.J. Ball, A. Dillenz, G. Busse, J.-C. Krapez, F. Galmiche and X. Maldague 'Round robin comparison2 Round Robin comparison II of the capabilities of various thermographic techniques in the detection of defects in carbon fibre composites' QIRT 2000 p.224

[6] J Rantala, D Wu, a Salerno and G Busse 'Lock-in thermography with mechanical loss angle heating at ultrasonic frequencies' QIRT 1996 p.389-393

[7] Th Zweschper, A Dillenz and G Busse 'Ultrasound lockin thermography - an NDT method for the inspection of Aerospace structures' Insight 2001 p.173-179

[8] M Bauer, Ch Guntrum, Mota, W rippel and G Busse 'Thermographic characterisation of defects and failure in polymer composites' QIRT 1992 p. 141-144

[9] X Han, E Yitamben, L D Favro, G Newaz, R L Thomas 'Acoustic chaos for enhanced detectability if cracks by sonic infrared imaging' Journal of Applied Physics 2004 p.3792-3797

[10] Z Ouyang, L D Favro, R L Thomas, X Han 'Theoretical modeling of thermosonic imaging of cracks' Review of Progress in Quantitative Nondestructive Evaluation, vol. 21, 2002 p.577-581

[11] H S Carslaw and J C Jaeger 'Heat conduction in solids' Oxford University press 1959

[12] W R Williams and M B Rankin 'Laser vibrometry and strain gauge measurements of thermosonic activation' Review of Quantitative Nondestructive Evaluation, vol 22, 2003, p.492-499

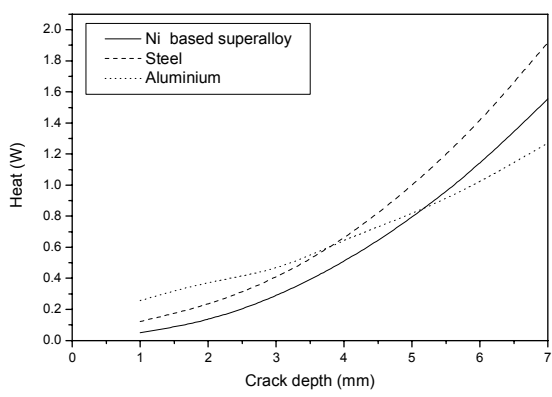

(a)

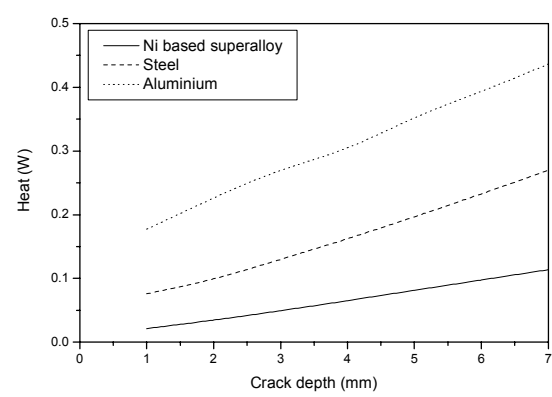

(b)

Fig. 1. The energy required to produce a $0.1^{\circ} \mathrm{C}$ surface temperature rise for a $10 \mathrm{~mm}$ long crack and a pulse time of $0.5 \mathrm{~s}$ for (a) heating at the crack tip only and

(b) heating over the sides of the crack 


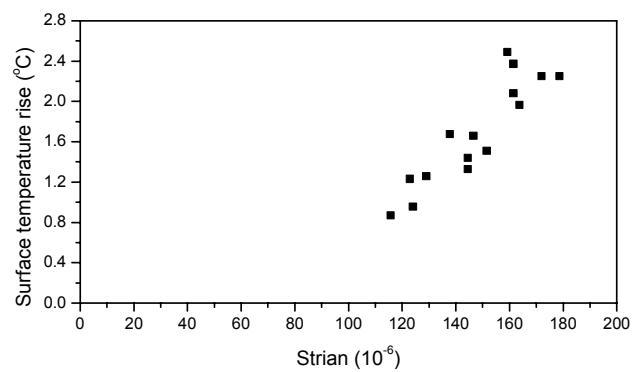

Fig. 2. The relationship between the measured strain and the surface temperature rise

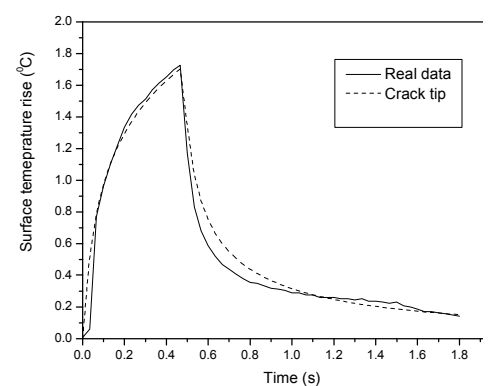

(a)

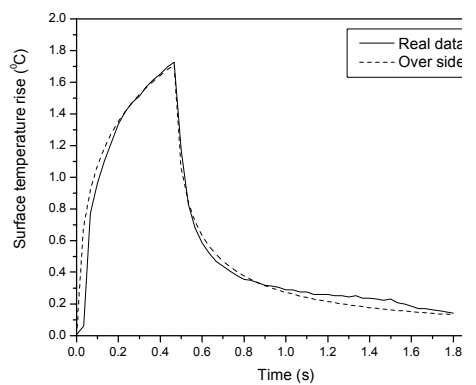

(b)

Fig. 3. Curve fitting modeled data to empirical data for (a) heating at the crack tip only and (b) heating over the sides of the crack

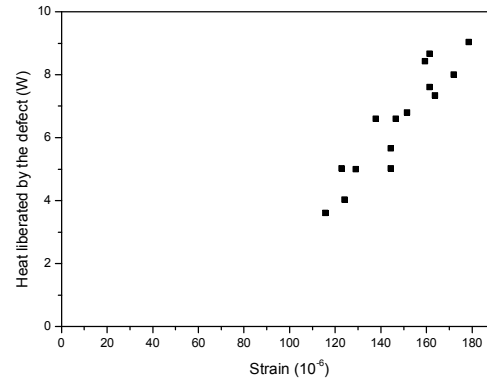

(a)

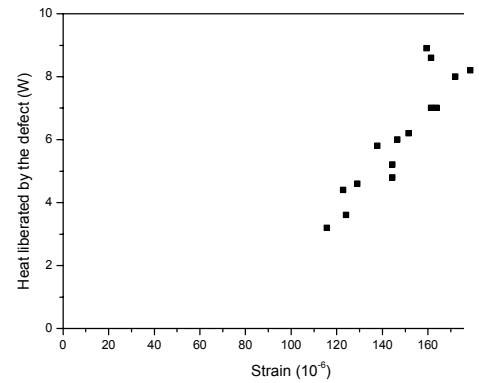

(b)

Fig. 4. The relationship between measured strain and the heat liberated at the defect for (a) heating at the crack tip only and (b) heating over the crack sides 